\title{
A manual-based family intervention for families living with the consequences of traumatic injury to the brain or spinal cord: a study protocol of a randomized controlled trial
}

\author{
Pernille Langer Soendergaard ${ }^{1,2^{*}}$ (D) Mia Moth Wolffbrandt ${ }^{1}$, Fin Biering-Sørensen ${ }^{3,4}$, Malin Nordin ${ }^{5}$, Trine Schow ${ }^{6}$, \\ Juan Carlos Arango-Lasprilla, $a^{7,9}$ and Anne Norup ${ }^{1,2}$
}

\begin{abstract}
Background: Acquiring a traumatic injury constitutes a severe life change for the survivor, but also for the surrounding family. The paradigm of helping the family has primarily been on psychosocial interventions targeting caregivers. However, interventions including both survivor and caregivers should be an essential part of treatment, as the whole family's functional level and mental health can be affected. The current study protocol presents a manualized family intervention for families living with traumatic injury to the brain (TBI) or spinal cord (tSCl). The objectives are to investigate if the intervention improves quality of life (QoL) and decreases burden. It is hypothesized that the family intervention improves problem-solving strategies and family dynamics, which will reduce the burden. This may improve the caregivers' mental health, which will improve the support to the survivor and QoL.

Methods: The study is an interventional, two-arm, randomized controlled trial. During a 2-year period, a total of 132 families will be included. Participants will be recruited from East-Denmark. Inclusion criteria are (1) TBI or tSCI, (2) $\geq 18$ years of age, (3) $\geq 6$ months to $\leq 2$ years since discharge from hospital, (4) ability to understand and read Danish, (5) cognitive abilities that enable participation, and (6) a minimum of one family member actively involved in the survivor's life. Exclusion criteria are (1) active substance abuse, (2) aphasia, (3) prior neurologic or psychiatric diagnose, and (4) history of violence. Within each disease group, families will be allocated randomly to participate in an intervention or a control group with a ratio 1:1. The intervention groups receive the family intervention consisting of eight sessions of 90 min duration. Families in the control groups receive $2 \mathrm{~h}$ of psychoeducation. All participants complete questionnaires on QoL, self-perceived burden, family dynamics, problem-solving strategies, mental health, and resilience at pre-intervention, post-intervention, and 6-month follow up.
\end{abstract}

Conclusion: If the intervention is found to have effect, the study will contribute with novel knowledge on the use of a manual-based intervention including the entire family. This would be of clinical interest and would help families living with the consequences of $\mathrm{TBI}$ or $\mathrm{tSCl}$.

(Continued on next page)

\footnotetext{
* Correspondence: Pernille.langer.soendergaard@regionh.dk

'Department of Neurorehabilitation, TBI Unit, Rigshospitalet, Kettegaard Allé

30, 2650 Hvidovre, Denmark

${ }^{2}$ Department of Psychology, University of Southern Denmark, Campusvej 55,

5230 Odense, Denmark

Full list of author information is available at the end of the article
}

(c) The Author(s). 2019, corrected publication December 2019. Open Access This article is distributed under the terms of the Creative Commons Attribution 4.0 International License (http://creativecommons.org/licenses/by/4.0/), which permits

unrestricted use, distribution, and reproduction in any medium, provided you give appropriate credit to the original author(s) and the source, provide a link to the Creative Commons license, and indicate if changes were made. The Creative Commons Public Domain Dedication waiver (http://creativecommons.org/publicdomain/zero/1.0/) applies to the data made available in this article, unless otherwise stated. 
(Continued from previous page)

Trials registration: ClinicalTrials.gov, NCT03814876. Retrospectively registered on 24 January 2019.

Keywords: Traumatic brain injury, Traumatic spinal cord injury, Family intervention, Randomized controlled trial, Study protocol, CONSORT-SPI 2018

\section{Background}

\section{Background and objectives}

Each year traumatic injury to the brain (TBI) or spinal cord (tSCI) affects approximately 3000 individuals and their families in Denmark [1, 2]. TBI and tSCI are complex injuries often followed by a broad range of disabilities. Survivors of TBI may experience cognitive, somatic, affective, behavioural, and motor difficulties [3, 4 , and survivors of tSCI may experience complete paralysis or motor and sensory difficulties in relation to urinary bladder, bowel management, sexual function, respiratory and cardiovascular function etc. [5]. Acquiring a traumatic injury not only has an impact on the survivor, but will also affect their families, as they will have to adapt to the changed life situation as well [6-8]. The consequences can affect the family early on $[9,10]$, but also long term [11-13]. Several families report low level of family functioning up to 5 years after injury $[14,15]$. Very often the family members must perform a considerable number of tasks related to the patient. About $43 \%$ of caregivers for TBI survivors report spending more than $5 \mathrm{~h}$ per week helping their injured family member, and of this group, $22 \%$ spend more than $16 \mathrm{~h}$ each week $[11,12]$. In a group of caregivers for patients with tSCI, $39 \%$ reported they stopped working to be able to take care of their injured family member [16]. Families with TBI report high frequencies of anxiety and depression symptoms [17, 18], and high emotional burden [7, 13]. Similar results have been found among families living with tSCI $[6,19]$. Furthermore, impaired quality of life (QoL) has been reported for both groups [5, 10, 12, 13, $16,20]$, for as long as up to 20 years after injury [21]. The reason may be that the family is facing a new life situation for which they are not prepared, as a traumatic injury is sudden and unexpected [22]. Consequently, most families do not have the appropriate coping strategies [23-25]. Thus, it is important to help the family in coping with the changed life situation [26].

So far, the paradigm and focus of helping the family has been psychosocial interventions primarily targeted at caregivers. The assumption behind most interventions has been that the best way to take care of the survivor is to take care of the caregiver. Thus, most interventions have included only the primary caregiver of the close family. Such interventions have consisted of psychoeducation and support [27-30], stress management [18, 31] skill-building and problem-solving strategies [32-35].
These interventions have shown improvement of problem-solving strategies, reduced emotional burden, anxiety and depression for the primary caregiver. However, it is unclear how this type of intervention affects the survivor. As most intervention programmes work with the survivor and caregiver separately, there is a lack of focus on the entire family. Interventions including the whole family should be an essential part of the treatment [36], as the injury can affect the family dynamics, including roles, boundaries, and communication. Both internationally [19, 24, 37-39] and nationally in Denmark [7, $9,11,40]$, negative effects on the family's functional level and mental and physical health have been welldocumented. This can affect the quality of the care that the caregiver provides [8]. By intervening in the functioning of the entire family, it may be possible to influence QoL and the outcome for both the survivor and the caregiver [8]. However, such a relationship has not yet been elucidated longitudinally, and there have been only a few cross-sectional studies [25, 41].

In a pilot study conducted in Latin America [42], the effectiveness of a newly developed eight-session manualized family intervention for individuals with spinal cord injury (SCI) was evaluated. The preliminary results of this pilot study showed large effect sizes in relation to depression, anxiety, stress and problem-solving strategies. Families, who received the intervention, experienced significant improvements in depression and anxiety, and this effect was maintained at 6-month follow up. Furthermore, effects on burden were also found as well as improved problem-solving strategies. Based on these promising pilot results, this current study seeks to investigate the family intervention in a Danish population.

Consequently, the aim of the present study is to investigate the effect of the eight-session manualized family intervention on QoL in individuals with TBI or tSCI and their family members, compared to a control group. The effect will be measured short term (post-intervention) and long term (6-month follow up). It is assumed that people who survive a traumatic injury do so in the context of their family. Therefore, a minimum of one family member, actively involved in the patient's life, must be included in each session of the intervention. To our knowledge, the present randomized controlled trial (RCT) will be the first to evaluate the effectiveness of a manual-based family intervention to improve QoL in the entire family. This will be investigated by the following 
primary research question: Will the families in the intervention groups report improved QoL compared to the families in the control groups? The secondary research question is: Will the families in the intervention groups report decreased burden compared to the families in the control groups? It is hypothesized that the family intervention will lead to improved QoL and decreased burden for both the survivor of TBI or tSCI and their caregivers. Consequently, better mental health of the caregiver will improve the care and support they provide to the survivor. This improvement will ultimately improve the QoL of the survivor.

\section{Methods}

Trial design

This is a two-arm RCT of a manualized family intervention conducted in two different disease groups: (1) patients with TBI and (2) patients with tSCI, participating with a minimum of one family member actively involved in the patient's life. The duration of the study is from October 2018 to October 2021 with an inclusion period of 2 years. The trial was developed according to the guidelines for Standard Protocol Items: Recommendations for Interventional Trials (SPIRIT) statement [43] (see Additional file 1 and Fig. 1), and will be reported as stated in the Consolidated Standards of Reporting Trials statement for reporting randomized trials of social and psychological interventions (CONSORT-SPI) [44].

\section{Participants}

The present study has an uptake area covering the eastern part of Denmark including the Region Zealand and Capital Region with a total of almost $46 \%$ of all inhabitants in Denmark [45]. Patients from this part of Denmark, who have either TBI (moderate to severe) or

\begin{tabular}{|c|c|c|c|c|c|}
\hline \multirow[b]{3}{*}{ TIMEPOINT } & \multicolumn{5}{|c|}{ STUDY PERIOD } \\
\hline & \multirow{2}{*}{$\begin{array}{c}\text { Pre-allocation } \\
-t_{1}\end{array}$} & \multicolumn{2}{|c|}{ Allocation/baseline } & \multicolumn{2}{|c|}{ Post-allocation } \\
\hline & & 0 & $t_{1}{ }^{*}$ & $t_{2}^{* *}$ & $t_{3}{ }^{\text {**** }}$ \\
\hline \multicolumn{6}{|l|}{ ENROLMENT: } \\
\hline \multicolumn{6}{|l|}{ Eligibility screen } \\
\hline Allocation & & & $x$ & & \\
\hline Informed consent & & & $x$ & & \\
\hline Randomization & & & $X$ & & \\
\hline \multicolumn{6}{|l|}{ INTERVENTIONS: } \\
\hline \multicolumn{6}{|l|}{$\begin{array}{r}\text { Intervention Group (a } \\
\text { manual-based family } \\
\text { intervention) }\end{array}$} \\
\hline \multicolumn{6}{|l|}{$\begin{array}{r}\text { Control Group } \\
\text { (psychoeducational } \\
\text { training) }\end{array}$} \\
\hline \multicolumn{6}{|l|}{ ASSESSMENTS: } \\
\hline $\begin{array}{r}\text { Register based } \\
\text { information }\end{array}$ & $X$ & & & & \\
\hline Telephone interview & & $X$ & & & \\
\hline $\begin{array}{r}\text { Demographic } \\
\text { information }\end{array}$ & & & $x$ & $X$ & $\mathrm{X}$ \\
\hline $\begin{array}{r}\text { Outcome variables } \\
\text { (Questionnaires) }\end{array}$ & & & $\mathrm{X}$ & $X$ & $X$ \\
\hline
\end{tabular}

* $t_{1}$ : Both the intervention group and control group complete questionnaires at $t_{1}$. From $t_{1}$ to $t_{2}$ families in the intervention group receive the family intervention. During these eight weeks, the families in the control group receive two hours of psychoeducational training.

${ }^{* *} t_{2}$ : Families in the intervention group and control group complete the same questionnaires at $t_{2}$. This timepoint is approximate eight weeks since $t_{1}$.

${ }_{* * *} t_{3}$ : Both the intervention group and control group complete the same questionnaires at $t_{3}$. The families receive the questionnaires in a stamped envelope and return it. This timepoint is 6 months since $t_{2}$.

Fig. 1 Standard Protocol Items: Recommendations for Interventional Trials (SPIRIT) table of enrolment, intervention, and assessments 
tSCI, can be included with a minimum of one family member if meeting the following criteria:

1. Age $\geq 18$ years at time of inclusion in the study

2. Between $\geq 6$ months and $\leq 2$ years since discharge from hospital

3. Ability to understand and read Danish

4. Patients with TBI must score $\geq 7$ on the Rancho Los Amigos Scale at time of inclusion in the study, indicating the resolution of post-traumatic amnesia (PTA)

5. Cognitive abilities that enable participation in a manualized intervention (when in doubt, the patient must have Mini Mental State Examination score $\geq 23$ )

Family members must meet the following criteria:

1. Actively involved in the patient's life

2. Age $\geq 18$ years at time of inclusion in the study

3. Ability to understand and read Danish

Patients and family members will be excluded if they meet any of the following criteria:

1. Active substance abuse

2. Severe aphasia

3. Prior diagnosis of neurologic or psychiatric disorder

4. History of violence in the family

\section{Recruitment and informed consent}

The research study will be conducted at the Department of Neurorehabilitation, TBI Unit, Rigshospitalet, Denmark. The participants will be recruited from two different clinics in Denmark: one clinic for patients with TBI and one clinic for patients with tSCI. Two project nurses will recruit participants, provide information about the study, randomize, and obtain written informed consent. All family members are informed that they have the right to withdraw their consent at any time during the study period. In the case of withdrawal, the family is asked if data already collected can be included in future analysis.

\section{Manualized family intervention}

In the present study, the manualized intervention "Traumatic Brain Injury (TBI)/Spinal Cord Injury (SCI) Family Intervention" will be used [46]. The whole manual for conducting the intervention has been published by the developers and can be accessed from the online version (doi:https://doi.org/10.1310/sci2201-49) of their pilot study in the supplementary material [42].

The intervention was developed for patients with TBI or $\mathrm{tSCI}$ and their families with the overall purpose to improve the functioning of the individual and the family.
The intervention was based on clinical experience and empirical research and constructed by a neuropsychologist in collaboration with a family therapist and a psychologist specializing in cognitive behavioural therapy [46]. In 2017, the manual was translated into Danish by authors TS and AN, and all questions concerning the intervention and translation process were discussed with the developers.

The intervention consists of a 90-min session once a week for 8 weeks and includes strategies and elements from both couples and family therapy. Session 1 is facilitated by the nurse and all participants (both intervention and control groups) will be supervised, while completing the questionnaires before randomization. Sessions 2-8 will address different topics or special strategies and are facilitated by a trained neuropsychologist. Between each session the participants will complete a homework assignment, a between-session task, where the new strategies can be practiced. The content of the sessions is structured as follows (Table 1) [42].

Each session of the intervention follows the same structure (except for session 1: Introduction). The session begins with a quote relevant for the topic of the session. Afterwards, the homework assignment will be evaluated. A new topic is then presented. New techniques and strategies will be practiced and used in relation to a specific challenge faced by the family. Finally, a new homework assignment will be presented.

The family intervention aims at helping families [46] to (1) share ideas about and experiences of the traumatic injury and to create a greater understanding of each other; (2) elicit misunderstandings about a traumatic injury; (3) change mindsets from negative to more positive aspects of their situation; (4) recognise the influence their thoughts have on their emotions; (5) identify signs that indicate escalation of emotions and to learn strategies to manage their emotions; (6) communicate more effectively; and (7) understand the importance of boundaries in the family and learn to clarify them together [46].

\section{Control group}

Families allocated to the control group will participate in one psychoeducational session of $2 \mathrm{~h}$, either individually or in a group. This will take place within the first 8 weeks after baseline. The facilitator will be an experienced neuropsychologist, who is not involved with the intervention groups. The content of the 2-h session will be equal for all participants in the control groups. They will receive information about the consequences of a traumatic injury, and how it can affect the entire family. Furthermore, information on normal emotional reactions, both in the acute phase, but also in long term, will be discussed. At the end of the session, the family members will be able to share their experiences, and the facilitator will give them advice about, where to receive 
Table 1 An overview of the sessions, the content and the frequency for the intervention group

\begin{tabular}{|c|c|c|c|}
\hline Week & Session (topic) & Topic of discussion & Facilitator \\
\hline 1 & Introduction & $\begin{array}{l}\text { Information about the study; consent to participation; } \\
\text { completion of baseline questionnaires; randomization; } \\
\text { The homework assignment is given ( } 90 \text { min) }\end{array}$ & $\begin{array}{l}\text { Nurse. If the family is randomized to the } \\
\text { intervention group at the end of the } \\
\text { session, the neuropsychologist will present } \\
\text { the homework assignment }\end{array}$ \\
\hline 2 & Making meaning & $\begin{array}{l}\text { Presentation of the study and the individual sessions; } \\
\text { expectations to the participants and the facilitator; } \\
\text { making meaning of TBI/tSCl and psychoeducation; the } \\
\text { homework assignment is reviewed and a new given; } \\
\text { session rating scale ( } 90 \mathrm{~min} \text { ) }\end{array}$ & Neuropsychologist \\
\hline 3 & Shifting focus & $\begin{array}{l}\text { The relationship between thoughts, feelings, and } \\
\text { behaviour; the homework assignment is reviewed } \\
\text { and a new given; session rating scale ( } 90 \text { min) }\end{array}$ & \\
\hline 4 & Managing emotions & $\begin{array}{l}\text { Learning to identify signs indicating an escalation of } \\
\text { emotions and techniques to handle emotions; strategies } \\
\text { for overcoming negative emotions; the homework } \\
\text { assignment is reviewed and a new given; session rating } \\
\text { scale ( } 90 \mathrm{~min} \text { ) }\end{array}$ & \\
\hline 5 & $\begin{array}{l}\text { Communicating } \\
\text { effectively }\end{array}$ & $\begin{array}{l}\text { Talk-listening techniques and communication improving } \\
\text { strategies; communication danger sings; the homework } \\
\text { assignment is reviewed and a new given; session rating } \\
\text { scale ( } 90 \mathrm{~min})\end{array}$ & \\
\hline 6 & Finding solutions & $\begin{array}{l}\text { From problem-talk to solution-talk; problem-solving } \\
\text { strategies; the homework assignment is reviewed and } \\
\text { a new given; session rating scale ( } 90 \text { min) }\end{array}$ & \\
\hline 7 & Boundary making & $\begin{array}{l}\text { Understanding the importance of boundaries in the family; } \\
\text { self-care; healthy family dynamics; the homework } \\
\text { assignment is reviewed and a new given; session rating } \\
\text { scale ( } 90 \mathrm{~min} \text { ) }\end{array}$ & \\
\hline 8 & Conclusion and farewell & $\begin{array}{l}\text { Summary of learned skills; the homework assignment is } \\
\text { reviewed; session rating scale; completion of post- } \\
\text { intervention questionnaires (the facilitator leaves the room) } \\
\text { (90 min) }\end{array}$ & \\
\hline $\begin{array}{l}34 \text { ( } 6 \text { months } \\
\text { after session 8) }\end{array}$ & Follow up & $\begin{array}{l}\text { Completion of the follow-up questionnaires. The families } \\
\text { receive the questionnaires in a stamped envelope. The } \\
\text { families return the questionnaires to the nurse. They will } \\
\text { receive a reminder if they forget to return the } \\
\text { questionnaires and a follow up by phone if no answer }\end{array}$ & Nurse \\
\hline
\end{tabular}

more support and/or information. The control groups are offered psychoeducational support as it seems unethical not to offer these families anything because of the promising results from the pilot study in Latin America [42]. The rationale behind offering these families a single psychoeducational session is that they will receive some support, but the support is expected to have a short-term effect and will consequently not affect the results when comparing the two groups (intervention group and control group). If the family intervention turns out to be effective, all families allocated to the control groups will be offered the 8-week family intervention after the study completion.

\section{Outcomes}

The manual-based family intervention for families with TBI or tSCI has not been implemented in Denmark prior to this study. Thus, we are interested in the effect of the family intervention, but also the families' subjective experiences of participating in the intervention. Consequently both quantitative and qualitative data will be collected.

\section{Questionnaires}

Socio-demographic data The following information will be collected at baseline (session 1): age, gender, number of years of education, and cause and date of injury. Other socio-demographic characteristics will be collected at all three timepoints, i.e. baseline, postintervention ( 8 weeks after baseline) and at 6-month follow up including civil status, family member relationship, duration of relationship, members in the household, employment status, number of weekly hours spent on work or education, comorbidities, medical drug use, social network, and whether they have participated or 
currently are participating in another therapeutic setting with a psychologist. The patients will also complete questions about rehabilitation, if they still receive support from an occupational or physio therapist, and if they have been hospitalized during the family intervention. Caregivers will complete information on the hours spent on caring/supervising the patient each day.

Injury characteristics The Glasgow Coma Scale [47] and length of PTA $[48,49]$ will be used as an indicator of injury severity for patients with TBI. The neurological level and severity of the spinal cord lesion will be assessed in patients with tSCI according to the International Standards for Neurological Classification of Spinal Cord Injury, including the American Spinal Injury Association Impairment Scale [50, 51]. The length of stay will be registered for both disease groups.

Primary and secondary outcome The effect of the family intervention will be measured by self-report questionnaires. The outcome measurements are outlined in Table 2 and will be measured at baseline, postintervention, and at 6-month follow up (Table 2).

All participants in the two groups will complete selfreport questionnaires to collect generic QoL data as the primary outcome measurement, and self-perceived burden, family dynamics, problem-solving strategies, mental health, and resilience as secondary outcome measurements. In cases where the family consists of a dyad, e.g. a married couple, mother and daughter etc., rather than several family members, the Relationship Assessment Scale (RAS) [63-65] will be completed as a supplement.

Treatment compliance All participants and the neuropsychologist will complete a measure of compliance. Participants allocated to the intervention group will individually complete the Session Rating Scale (SRS) [67] after each session. The SRS is a paper and pencil four-item scale used to evaluate the individuals' experience with the relevance of the specific topic of the session, including strengths and weaknesses, and their experience with the neuropsychologist. The neuropsychologist will leave the room during this task. The families will be instructed to put the completed questionnaire in an envelope, consequently the neuropsychologist will be blinded to their answers.

When the 8 sessions are completed, the neuropsychologist will complete an evaluation form, the Therapist Checklist Scale, which evaluates the participants' attendance [68]. It is a five-item scale, where the neuropsychologist will rate the participants according to their engagement in the intervention, including level of participation, homework assignments, interaction with the other family members and the neuropsychologist, and their ability to use the strategies from each session $[68,69]$.

\section{Semi-structured interviews}

The experiences with the manual-based family intervention will be investigated qualitatively by conducting individual semi-structured interviews with 20 families who

Table 2 Primary and secondary outcome measures

\begin{tabular}{|c|c|c|c|}
\hline Domain & Assessment & Test tool (outcome measure) & Administered by \\
\hline \multirow[t]{3}{*}{ Quality of life (QoL) } & Generic QoL & Short Form-36 (SF-36) $[9,10,52,53]$ & All participants \\
\hline & \multirow[t]{2}{*}{ The patient's disease-specific QoL } & QoL after Brain Injury (Qolibri) [54] & Participants with TBI \\
\hline & & $\begin{array}{l}\text { International Spinal Cord Injury QoL } \\
\text { Basic Data Set [55] }\end{array}$ & Participants with $\mathrm{tSCl}$ \\
\hline \multirow[t]{2}{*}{ Self-perceived burden } & $\begin{array}{l}\text { General burden, isolation, disappointment, } \\
\text { emotional involvement, environment }\end{array}$ & Caregiver Burden Scale (CBS) $[12,56]$ & Caregivers \\
\hline & Physical, emotional and financial burden & Self-Perceived Burden Scale (SPB) [57] & Participants with $\mathrm{TBI}$ or $\mathrm{tSCl}$ \\
\hline \multirow[t]{3}{*}{ Family dynamic } & Flexibility & \multirow{3}{*}{$\begin{array}{l}\text { Family Adaptability and Cohesion } \\
\text { Evaluation Scales-IV (FACES-IV) [58] }\end{array}$} & \multirow[t]{3}{*}{ All participants } \\
\hline & Cohesion & & \\
\hline & Communication & & \\
\hline \multirow[t]{3}{*}{ Problem-solving abilities } & Confidence & \multirow[t]{3}{*}{ Problem-Solving Inventory (PSI) [59] } & \multirow[t]{3}{*}{ All participants } \\
\hline & Personal control & & \\
\hline & Approach-avoidance & & \\
\hline \multirow[t]{3}{*}{ Mental health } & Depression & Patient Health Questionnaire-9 (PHQ-9) [60] & \multirow[t]{3}{*}{ All participants } \\
\hline & Anxiety & Generalized Anxiety Disorder-7 (GAD-7) [61] & \\
\hline & Satisfaction with life & Satisfaction with Life Scale (SWLS) [62] & \\
\hline Relationship & Relationship to relatives & Relationship Assessment Scale (RAS) [63-65] & All participants if a dyad \\
\hline Resilience & Resilience and robustness & The Resilience Scale for Adults (RSA) [66] & All participants \\
\hline
\end{tabular}


have finished the 8-week family intervention. The families will be selected based on representativeness of disease (TBI or tSCI), different family structures (e.g. couples, parents with adult children, siblings), and age and gender of the survivor. Qualitative data will be used to analyse the families' individual experience of participating in the intervention. The interviews will include the families' experiences of the structure and content of each session, and the amount and content of homework. Furthermore, the participants will be asked if and how the intervention was meaningful for their specific situation. A research nurse will conduct the interviews within the first 4 weeks after the intervention has been completed.

\section{Sample size}

Preliminary statistical power calculations have been carried out to allow us to detect any group effect (intervention versus control in the TBI and tSCI group, respectively) on the primary outcome variable, i.e. generic QoL data assessed by the Short Form-36 (SF-36) [9, 10]. Numbers of participants required in the study were calculated based on the Mental Component Summary (MCS) of the SF-36 [52, 53]. As no studies have been carried out in Denmark using the SF-36 in patients with a traumatic injury, a Norwegian study [70] was used for the sample size calculations. In the Norwegian study, a score of 43.8 (SD 12.5) was reported among patients with moderate to severe TBI. Based on a confidence level of $95 \%$, a power level of $80 \%$, and a significant difference of 5 points between the intervention and control group, it will be necessary to recruit 182 participants to each arm of the trial i.e. the TBI and tSCI arms. To account for a $10 \%$ drop-out rate, 400 participants in total must be included.

\section{Randomization}

\section{Sequence generation and allocation concealment} mechanism

Within each disease group, families will be allocated randomly to the intervention group or the control group with an allocation ratio of 1:1. The Sealed Envelope application will be used to generate random allocation sequences: this is an online software application for randomizing patients into clinical trials [71]. The random allocation sequence will be generated and concealed from the employees in the project. The families will consecutively be allocated to each group. Two arms for the TBI group and two arms for the tSCI group will be created with 66 families in each disease-specific group. The randomization will be conducted in blocks of 22, which increases the likelihood that the intervention groups and the control groups will have the exact same number of participants, even in the case of recruiting fewer participants than expected. When entering the unique family ID number in Sealed Envelope, the application randomizes the family to either the intervention group or the control group.

\section{Implementation and awareness of assignment}

The project nurses will be responsible for identifying and recruiting families that meet the inclusion criteria. The random allocation sequence will be generated by the nurses using the online randomization procedure [71]. Families will complete the baseline questionnaires before randomization.

Because of the nature of the study, it will be clear to all participants and the neuropsychologist whether the families are allocated to the intervention group or the control group. It is not possible to blind the participants or the neuropsychologist to the group allocation. The nurses will collect the study data. The neuropsychologist and statistician performing the data analyses will not be involved in collection of data on the outcome measures. Thus, they will be blinded to the association between the data from the questionnaires and the group to which the families are allocated. The nurses who are responsible for the data collection will not participate in data analysis.

\section{Analytical methods}

Data will be stored in a password-protected electronic database, REDCap [72], which is a web-application for building and managing databases. All participants will be assigned a unique trial ID number and all demographic data, and data from the files and questionnaires will be stored in the database to ensure quality of the data. The nurses will be responsible for this part of the data management process.

The following analyses will be conducted in each arm of the trial. First, the baseline level of the primary outcome, generic QoL data, will be compared between groups using one-way analysis of variance (ANOVA). If there are significant differences at baseline, we will adjust for these in the following analyses. Intention-to treat (ITT) analyses will be performed to examine the effect of the family intervention using repeated measures ANOVA (rANOVA), with "time" as the repeated measurement. The comparison between the intervention group and control group in each disease group will be the between-group factor. Baseline characteristics will be assessed and compared between groups using one-way ANOVA, and we will adjust for these characteristics if necessary.

Little's Missing Completely at Random (MCAR) approach will be applied for analysis of group allocation and of disease group to investigate whether drop-outs or 
missing data are associated with one of these parameters. Data analysis will be completed using STATA.

\section{Discussion}

The objective is to investigate the effectiveness of the Danish version of the manualized family intervention for families living with TBI or tSCI. The project is innovative as it includes all family members in the intervention. The randomized controlled design will provide knowledge about the effectiveness of the intervention, and furthermore the semi-structured interviews will give insight into the individual experiences of the participating families. This study will provide contributions to the literature on the importance of including the whole family in the intervention. The manual-based family intervention addresses the family function, including both the survivor of TBI or tSCI and the closest family members.

If the intervention is evaluated as having an effect, the aim is to make the family intervention a permanent programme. If we identify an effect of the manual-based family intervention on the primary outcome measure, the aim is to strengthen efforts towards helping patients in these disease groups and their families in Denmark. This can involve cross-sectoral partnerships between the hospital departments and specific municipalities in Denmark, and can include training of professionals working in rehabilitation. With this project, the aim is to ensure that survivors of a traumatic injury and their families will be helped to regain the structure and function in the family, and consequently experience improved health.

\section{Limitations and generalizability}

The present study will have some limitations that should be addressed. First, the study will only include families, who are willing to participate. Consequently, some families, who would fulfill our inclusion criteria, will not be included. One could speculate which families will be willing to participate in the study. Will it be families, who need help and support, or families, who have the time and resources? If a family refuses to participate, the reason for refusal will be obtained. Furthermore, as participation will be voluntary, all family members have the right to withdraw their consent if they want to quit the project. One could speculate whether families allocated to the control group will be more likely to drop out compared to families in the intervention group, because of a great desire to receive help. However, as the family intervention is very intense and time-consuming, this can also affect the risk of drop-outs in the intervention group. Therefore, prior to the allocation the nurses will inform the families about the content of the intervention in both groups both orally and in writing.
Second, most patients included in the study will have been hospitalized for specialized neurorehabilitation due to a severe traumatic injury. However, patients with severe cognitive disabilities or severe aphasia will be excluded, as patients with such disabilities will not be able to participate fully in the family intervention. This will affect the generalizability of the study, as patients with the most severe injuries or sequela will be excluded.

Third, participants will be recruited from only two clinics in Denmark - one clinic for patients with TBI and one clinic for patients with tSCI. This warrants caution with respect to generalizing the results and could potentially limit the external validity. However, as the two clinics have an uptake area covering the whole eastern part of Denmark with a total of almost 2.6 million inhabitants [45], the representativeness and generalizability is expanded.

Finally, due to the study design, it will not be possible to blind the participants or the neuropsychologist to the allocation. However, the researchers responsible for statistically analysing baseline, post-intervention, and follow-up questionnaire data will not know to which group the families were allocated.

\section{Interpretation}

For families allocated to the intervention group, there can be some benefits and harms to consider. Participating in the family intervention may be costly for some families in terms of resources and time rather than an opportunity to receive support and help. This may influence the willingness to participate in the 8week intervention, as participation may seem too time-consuming. To minimize the burden for the families, the neuropsychologist and nurses will be flexible in scheduling the sessions and the location of the intervention, e.g. if the family members have work or study obligations, then it will be possible to schedule a late session, and if the patient suffers from severe fatigue, then the intervention can be offered at home. Furthermore, the families can have transport expenses reimbursed and the intervention is free of charge.

For families allocated to the control group, there can be some ethical considerations. All families are carefully informed about the study design prior to inclusion, including the information that the randomization to either the intervention group or the control group is 50:50. However, it is possible that families who are willing to participate in the project hope to be allocated to the intervention group. In the worst case, families allocated to the control group may feel rejected, as they had high hopes of receiving help and support. This may harm the family and make them withdraw their consent and quit 
the project. However, the families allocated to the control group will be offered one psychoeducational session with a neuropsychologist.

\section{Important information Registration}

ClinicalTrials.gov Identifier: NCT03814876, registered on 24 January 2019.

\section{Protocol}

The Protocol Record 2018_0004, Family Intervention Following Traumatic Injury is accessible to the public on ClinicalTrials.gov.

\section{Declaration}

The project is funded by The Council of Danish Victims Fund (grant 18-610-00024) and Foreningen Oestifterne (j.nr. 18-077). The funders have no influence on the design of the study, on data collection or analysis, and did not participate in the writing of the manuscript. The authors declare no other potential interests.

\section{Stakeholder involvement}

Stakeholders in the project are amongst others the clinics from where participants will be recruited. Furthermore, the patient organizations in Denmark may have an interest in the results of the project. However, the clinics and the patient organizations will not be involved in the trial design, conduct or analyses, and there will be no incentives offered.

Juan Carlos Arango-Lasprilla (JCAL) is one of the developers of the manual-based family intervention for families living with TBI or tSCI. He is a part of this research group, currently investigating the effect of the Danish version of the manualized family intervention.

\section{Trial status}

This is study protocol version 1.0. Recruitment of participants for the study commenced in September 2018, and the first family was included and participated in session 1 on 17 October 2018. At present, 13 families have been randomly assigned (8 families of a patient with TBI and 5 families of a patient with tSCI): 5 families have been randomized to the intervention group and 8 families have been randomized to the control group; currently, 4 families have participated in the semi-structured interview. The estimated enrollment period, including recruitment and inclusion, is anticipated to be 2 years (October 2020) and will conclude, when the estimated sample size has been included.

\section{Supplementary information}

Supplementary information accompanies this paper at https://doi.org/10. 1186/s13063-019-3794-5.

Additional file 1. SPIRIT 2013 Checklist: Recommended items to address in a clinical trial protocol and related documents.

\section{Acknowledgements}

We thank all families for their contributions to this study.

\section{Authors' contributions}

The manual-based family intervention was developed by JCAL. AN and TS translated the manual into Danish and made some cultural adjustments approved by JCAL. AN received the funding for the study and reported the study to the Danish Data Protection Agency and The Committees on Health Research Ethics in the Capital Region of Denmark. AN performed the initial statistical power analysis and generated the random allocation sequence. AN, TS, and PLS chose and, if necessary, translated the questionnaires into Danish. FBS, MN, MMW, and PLS screen all patients with TBI and tSCI for eligibility. MMW and MN recruit the families. PLS facilitate the intervention to the families allocated to the intervention group. PLS wrote the first draft of the manuscript and AN read the first draft and made initial revisions. All authors wrote sections, contributed to manuscript revision, and read and approved the submitted version.

\section{Funding}

The study is financed by The Council of Danish Victims Fund (grant 18-61000024), and by the Foreningen Oestifterne (j.nr. 18-077). The funders had no influence or role in the design of the study, collection, analysis, or interpretation of data, nor in the writing of the manuscript.

\section{Availability of data and materials}

The data materials are available from the corresponding author on reasonable request if the Danish Data Protection Agency accepts such request.

\section{Ethics approval and consent to participate}

The study is conducted in concordance with the Helsinki Declaration. Patients and family members will be informed orally and in writing about the purpose of the study before providing consent. Participation is voluntary. Data will be presented in anonymous form without any possibility to recognize the individual participants. The study has been reported to the Danish Data Protection Agency (journal no. VD-2018-159, I-Suite no. 6401). The Committees on Health Research Ethics in the Capital Region of Denmark, has stated that the study does not need their approval (journal no. H-18014858). All data will be handled according to the legislation of the Data Protection Agency.

Consent for publication

Not applicable.

\section{Competing interests}

The authors declare that they have no competing interests.

\section{Author details}

${ }^{1}$ Department of Neurorehabilitation, TBI Unit, Rigshospitalet, Kettegaard Allé 30, 2650 Hvidovre, Denmark. ²Department of Psychology, University of Southern Denmark, Campusvej 55, 5230 Odense, Denmark. ${ }^{3}$ Clinic for Spinal Cord Injuries, Rigshospitalet, University of Copenhagen, Havnevej 25, 3100 Hornbæk, Denmark. ${ }^{4}$ Institute for Clinical Medicine, University of Copenhagen, Blegdamsvej 3B, 2200 Copenhagen, Denmark. ${ }^{5}$ Department of Neurosurgery, Karolinska University Hospital, Eugeniavägen 3, 17176 Stockholm, Sweden. ${ }^{6}$ Research and Development, Brain Injury Center BOMI, Maglegaardsvej 15, 4000 Roskilde, Denmark. ${ }^{7}$ BioCruces Vizcaya Health Research Institute, Cruces University Hospital, Barakaldo, Spain. ${ }^{8}$ IKERBASQUE, Basque Foundation for Science, Bilbao, Spain. ${ }^{9}$ Department of Cell Biology, University of the Basque Country (UPV/EHU), Leioa, Spain. 
Received: 26 April 2019 Accepted: 10 October 2019

Published online: 27 November 2019

\section{References}

1. Noe BB, Mikkelsen EM, Hansen RM, Thygesen M, Hagen EM. Incidence of traumatic spinal cord injury in Denmark, 1990-2012: a hospital-based study. Spinal Cord. 2015;53(6):436-40.

2. Sundhedsstyrelsen, Sundhedsdokumentation. Hjerneskaderehabilitering en medicinsk teknologivurdering [Brain injury rehabilitation - a medical technology assessment]. København: Sundhedsstyrelsen, Sundhedsdokumentation, 2011. Serienavn 2011;13(1).

3. Anke A, Andelic N, Skandsen T, Knoph R, Ader T, Manskow U, et al. Functional recovery and life satisfaction in the first year after severe traumatic brain injury: a prospective multicenter study of a Norwegian national cohort. J Head Trauma Rehabil. 2015;30(4):38-49.

4. Andelic N, Arango-Lasprilla JC, Perrin PB, Sigurdardottir S, Lu J, Landa LO, et al. Modeling of community integration trajectories in the first five years after traumatic brain injury. J Neurotrauma. 2016;33(1):95-100.

5. Jain NB, Ayers GD, Peterson EN, Harris MB, Morse L, Connor KCO, et al. Traumatic spinal cord injury in the United States, 1993-2012. JAMA. 2015; 313(22):2236-43.

6. Scholten EWM, Kieftenbelt A, Hillebregt CF, De Groot S, Ketelaar M, Vissermeily JMA, et al. Provided support, caregiver burden and well-being in partners of persons with spinal cord injury 5 years after discharge from first inpatient rehabilitation. Spinal Cord. 2018;55(8):436-46.

7. Norup A, Petersen J, Mortensen EL. Relatives of patients with severe brain injury: growth curve analysis of anxiety and depression the first year after injury. Brain Inj. 2015;29(7-8):822-9.

8. Norup A. Family matters in neurorehabilitation: why, when, who, and how? Rev Iberoam Neuropsicol. 2018;1(1):17-31.

9. Norup A, Siert L, Mortensen EL. Emotional distress and quality of life in relatives of patients with severe brain injury: the first month after injury. Brain i. 2010;24(2):81-8.

10. Norup A, Welling K, Qvist J, Siert L, Mortensen L. Depression, anxiety and quality-of-life among relatives of patients with severe brain injury: the acute phase. Brain Inj. 2012;26(10):1-9.

11. Doser K, Norup A. Family needs in the chronic phase after severe brain injury in Denmark. Brain Inj. 2014;28(10):1230-7.

12. Doser K, Norup A. Caregiver burden in Danish family members of patients with severe brain injury: the chronic phase. Brain Inj. 2016;30(3):334-42.

13. Norup A, Snipes DJ, Siert L, Mortensen EL, Perrin PB, Arango-lasprilla JC. Longitudinal trajectories of health related quality of life in Danish family members of individuals with severe brain injury. Aust J Rehabil Couns. 2013;19(2):71-83.

14. Schönberger M, Ponsford J, Olver J, Ponsford M. A longitudinal study of family functioning after TBI and relatives' emotional status. Neuropsychol Rehabil. 2010;20(6):813-29.

15. Ponsford J, Olver J, Ponsford M, Nelms R. Long-term adjustment of families following traumatic brain injury where comprehensive rehabilitation has been provided. Brain Inj. 2003;17(6):453-68.

16. Nogueira PC, Rabeh S, Caliri M, Dantas S, Haas V. Burden of care and its impact on health-related quality of life of caregivers of individuals with spinal cord injury. Rev Lat Am Enferm. 2012;20(6):1048-56.

17. Ergh TC, Rapport LJ, Coleman R, Hanks R. Predictors of caregiver and family functioning following traumatic brain injury: social support moderates caregiver distress. J Head Trauma Rehabil. 2002;17(2):155-74.

18. Backhaus SL, Ibarra SL, Klyce D, Trexler LE, Malec JF. Brain Injury Coping Skills Group: A preventative intervention for patients with brain injury and their caregivers. Arch Phys Med Rehabil. 2010;91(6):840-8.

19. Arango-lasprilla JC, Plaza O, Drew A, Romero J, Pizarro J, Francis K, et al. Family needs and psychosocial functioning of caregivers of individuals with spinal cord injury from Colombia, South America. NeuroRehabilitation. 2010; 27(1):83-93.

20. Kolaskowsky-Hayner S, Miner K, Kreutzer J. Long-term life quality and family needs following traumatic brain injury. J Head Trauma Rehabil. 2001;16(4):374-85.

21. Nestvold K, Stavem K. Determinants of health-related quality of life 22 years after hospitalization for traumatic brain injury. Brain Inj. 2009;23(1):15-21.

22. Norup A. Severe brain injury: impact of family members in the early phases of rehabilitation. Glostrup: Copenhagen University Hospital; 2012.

23. Norup A, Siert L, Mortensen EL. Coping strategies, emotional distress and quality of life in relatives of patients with severe brain injury in Denmark: one year after injury. Aust J Rehabil Couns. 2013;19(2):142-54.
24. Norup A, Perrin PB, Cuberos-urbano G, Anke A, Andelic N, Doyle ST, et al. Family needs after brain injury: a cross cultural study. NeuroRehabilitation. 2015;36(2):203-14.

25. Lehan T, Arango-lasprilla JC, De Los RC, Quijano M. The ties that bind: The relationship between caregiver burden and the neuropsychological functioning of TBI survivors. NeuroRehabilitation. 2012;30(1):87-95.

26. Baker A, Barker S, Sampson A, Martin C. Caregiver outcomes and interventions: a systematic scoping review of the traumatic brain injury and spinal cord injury literature. Clin Rehabil. 2017;31(1):45-60.

27. Norup A, Siert L, Mortensen EL. Neuropsychological intervention in the acute phase: a pilot study of emotional wellbeing of relatives of patients with severe brain injury. J Rehabil Med. 2013;45:827-34.

28. Brown R, Pain K, Berwald C, Hirschi P, Delebanty R, Miller H. Distance education and caregiver Support Groups: Comparison of Traditional and Telephone Groups. J Head Trauma Rehabil. 1999;14(3):257-68.

29. Sanguinetti M, Catanzaro M. A comparison of discharge teaching on the consequences of brain injury. J Neurosci Nurs. 1987;19(5):271-5.

30. Carnevale G, Anselmi V, Busichio K, Millis S. Changes in ratings of caregiver burden following a management program for persons with traumatic brain injury. J Head Trauma Rehabil. 2002;17(2):83-95.

31. Singer G, Glang A, Nixon C, Cooley E, Kerns K, Williams D, et al. A comparison of two psychological interventions for parents and children with acquired brain injury: an exploratory study. J Head Trauma Rehabil. 1994;9(4):38-49.

32. Kreitzer N, Kurowski BG, Bakas T. Systematic review of caregiver and dyad interventions after adult traumatic brain injury. Arch Phys Med Rehabil. 2018;99(11):2342-54.

33. Rivera P, Elliott TR, Berry JW, Grant JS, Oswald K. Predictors of caregiver depression among community-residing families living with traumatic brain injury. NeuroRehabilitation. 2007;22(1):3-8.

34. Sethi AA, Nordestgaard BG, Agerholm-larsen B, Frandsen E, Jensen G, Tybjærg-hansen A. Angiotensinogen polymorphisms and elevated blood pressure in the general population The Copenhagen City Heart Study. Hypertension. 2001;37(3):875-81.

35. Sander AM, Clark AN, Atchison TB, Rueda M. A web-based videoconferencing approach to training caregivers in rural areas to compensate for problems related to traumatic brain injury. J Head Trauma Rehabil. 2009;24(4):248-61.

36. McDaniels SH, Hepworth J, Doherty WJ. Medical family therapy: a biopsychosocial approach to families with health problems. New York: Basic Book; 1992

37. Groom KN, Shaw TG, O'Connor ME, Howard NI, Pickens A. Neurobehavioral symptoms and family functioning in traumatically brain-injured adults. ArchClinNeuropsychol. 1998;13(8):695-711.

38. Gervasio A, Kreutzer J. Kinship and family members' psychological distress after traumatic brain injury: a large sample study. J Head Trauma Rehabil. 1997;12(3):14-26.

39. Manskow US, Sigurdardottir S, Andelic N, Skandsen T, Anke A. Factors affecting caregiver burden 1 year after severe traumatic brain injury : a prospective nationwide multicenter study. J Head Trauma Rehabil. 2015; 30(6):411-23.

40. Trujillo M, Perrin PB, Doser K, Norup A. Using personality traits to construct linear growth models using personality traits to construct linear growth models of mental health in family members of individuals with severe brain injury. Rehabil Psychol. 2016;61(4):389-96.

41. Lehan TJ, Stevens L, Arango-lasprilla JC, Días Sosa D, Espinosa Jl. Balancing act: the influence of adaptability and cohesion on satisfaction and communication in families facing TBI in Mexico. NeuroRehabilitation. 2012; 30(1):75-86.

42. Stevens LF, Lehan T, Angélica M, Durán S, Plaza O, Arango-lasprilla JC. Pilot study of a newly developed intervention for families facing serious injury. Top Spinal Cord Inj Rehabil. 2016;21(1):49-59.

43. Chan A, Tetzlaff JM, Altman DG, Laupacis A, Gøtzche P, Krleza-Jeric K, et al. SPIRIT 2013 statement: defining standard protocol items for clinical trials. Ann Intern Med. 2013;158(3):200-7.

44. Montgomery P, Grant S, Mayo-wilson E, Macdonald G, Michie S, Hopewell S, et al. Reporting randomised trials of social and psychological interventions: the CONSORT-SPI 2018 Extension. Trials. 2018;19(1):1-14.

45. Folketal i de enkelte kommuner og regioner 2017 [Number of citizens in each municipalities and regions 2017] [Internet]. 2017. Available from: https://www.dst.dk/pukora/epub/upload/22259/headword/dk/6.pdf 
46. Lehan T, Stevens LF, Arango-Lasprilla JC. Traumatic brain injury (TBI)/spinal cord injury (SCI) family intervention. Spinal Cord Inj Rehabil. 2016;22(1):49-59.

47. Teasdale $G$, Jennett B. Assessment and prognosis of coma after head-injury. Acta Neurochir. 1976:34(1-4):45-55.

48. li A, Kaste M, Sarna S. Predicting late outcome for patients with traumatic brain injury referred to a rehabilitation programme: a study of 508 Finnish patients 5 years or more after injury. Brain Inj. 1998;12(2):95-107 Available from: http:// www.tandfonline.com/action/journallnformation?journalCode=ibij20.

49. Walker WC, Stromberg KA, McCauley SR, Sima AP, Agyemang AA, Graham $\mathrm{KM}$, et al. Predicting long-term global outcome after traumatic brain injury: development of a practical prognostic tool using the traumatic brain injury model systems national database. J Neurotrauma. 2018;35(14):1587-95.

50. Kirshblum SC, Burns SP, Biering-sorensen F, Donovan W, Graves DE, Jha A, et al. International standards for neurological classification of spinal cord injury (Revised 2011). Spinal Cord Med. 2011;34(6):535-46.

51. Biering-Sørensen F, Devivo MJ, Charlifue S, Chen Y, New PW, Noonan V, et al. International Spinal Cord Injury Core Data Set (version 2 . 0) including standardization of reporting. Spinal Cord. 2017;55(8):759-64.

52. Bjorner J, Damsgård M, Watt T, Bech P. Danish manual for SF-36. Copenhagen: A health-related questionnaire. Medif; 1997.

53. Ware J, Sherbourne C. The MOS 36-Item Short-Form Health Survey ( SF-36 ): I. Conceptual framework and item selection. Med Care. 1992;30(6):473-83.

54. Von Steinbuechel N, Wilson L, Gibbons $H$, Muehlan $H$, Schmidt $H$, Schmidt S, et al. QOLIBRI overall scale: a brief index of health-related quality of life after traumatic brain injury. J Neurol Neurosurg Psychiatry. 2012:83:1041-7.

55. Charlifue S, Post MW, Biering-Sørensen F, Catz A, Dijkers M, Geyh S, et al. international spinal cord injury quality of life basic data set. Spinal Cord. 2012;50(3):672-5.

56. Elmståhl S, Malmberg B, Annerstedt L. Caregiver's burden of patients 3 years after stroke assessed by a novel caregiver burden scale. Arch Phys Med Rehabil. 1996;77(1):77-82.

57. Cousineau N, Mcdowell I, Hotz S, Hébert P. Measuring chronic patients' feelings of being a burden to their caregivers: developmental and preliminary validation of a scale. Med Care. 2003:41(1):110-8.

58. Olson D. FACES IV and the Circumplex Model: validation study. J Marital Fam Ther 2011:37(1):64-80.

59. Heppner P. The Problem Solving Inventory (PSI) Manual. Palo Alto: Consulting; 1988.

60. Kroenke K, Spitzer RL, Williams JBW. The PHQ-9: validity of a brief depression severity measure. JGIM. 2001;16(9):606-13.

61. Spitzer RL, Kroenke K, Williams JBW, Lowe B. A brief measure for assessing generalized anxiety disorder: The GAD-7. Arch Intern Med. 2006;166(5): 1092-7.

62. Diener E, Emmons R, Larsen R, Griffin S. The Satisfaction With Life Scale. J Pers Assess. 1985;49(1):71-5.

63. Rask M, Malm D, Kristofferzon M, Roxberg A, Svedberg P, Arenhall E, et al. Validity and reliability of a Swedish version of the Relationship Assessment Scale ( RAS ): a pilot study. Can J Cardiovasc Nurs. 2010;20(1):16-21.

64. Hendrick S, Dicke A, Hendrick C. The relationship assessment scale. J Soc Pers Relat. 1998;15(1):137-42.

65. Hendrick S. A generic measure of relationship satisfaction. J Marriage Fam. 1998;50(2):93-8.

66. Friborg $\mathrm{O}$, Hjemdal $\mathrm{O}$, Rosenvinge JANH, Martinussen $\mathrm{M}$. A new rating scale for adult resilience: what are the central protective resources behind healthy adjustment? Int J Methods Psychiatr Res. 1998;12(2):65-76.

67. Duncan BL, Miller SD, Sparks JA, Claud DA, Reynolds LR, Johnson LD. The Session Rating Scale: preliminary psychometric properties of a "working" alliance measure. J Br Ther. 2003;3(1):3-12.

68. Cantor J, Ashman T, Connor KD, Dijkers MP, Gordon W, Spielman L, et al. Evaluation of the Short-Term Executive Plus Intervention for executive dysfunction after traumatic Brain injury: a randomized controlled trial with minimization. Arch Phys Med Rehabil. 2014:95(1):1-9.

69. Howe El, Løvstad M, Langlo KS, Hellstrøm T, Spjelkavik Ø, Ugelstad H, et al. Feasibility of a cognitive rehabilitation program for individuals with mild-tomoderate traumatic brain injury: participants' engagement and satisfaction. Cogent Med. 2019;6(1):1-16.

70. Forslund M, Roe C, Sigurdardottir S, Andelic N. Predicting health-related quality of life 2 years after moderate-to-severe traumatic brain injury. Acta Neurol Scand. 2013;128(4):220-7.

71. Sealed Envelope [Internet]. Available from: https://www.sealedenvelope. com/
72. Harris PA, Taylor R, Thielke R, Payne J, Gonzalez N, Conde JG. Research electronic data capture (REDCap ) - a metadata-driven methodology and workflow process for providing translational research informatics support. J Biomed Inform. 2009;42(2):377-81.

\section{Publisher's Note}

Springer Nature remains neutral with regard to jurisdictional claims in published maps and institutional affiliations.

\section{Ready to submit your research? Choose BMC and benefit from:}

- fast, convenient online submission

- thorough peer review by experienced researchers in your field

- rapid publication on acceptance

- support for research data, including large and complex data types

- gold Open Access which fosters wider collaboration and increased citations

- maximum visibility for your research: over $100 \mathrm{M}$ website views per year

At BMC, research is always in progress.

Learn more biomedcentral.com/submissions 